Results IL-6 and CRP increased post-operatively peaking at $6 \mathrm{hrs}$ and $24 \mathrm{hrs}$ respectively however there was no statistically significant difference between the increase for BAL and non-BAL patients $(p>0.05)$. There was no significant increase or variation between the groups for IL-2, IL-4, IL-10, TNF- $\alpha$ or IFN- $\gamma(p>0.05)$. eNO tended to decrease in the BAL group and increase in the nonBAL group at 6 hrs although there was no significant difference between the groups $(p=0.167)$. Post-operative CXR atelectasis developed in 3 patients (2 BAL). One patient in each group developed SIRS.

Conclusion BAL has minimal impact on acute inflammation following bronchoscopy and mediastinoscopy. It may therefore be used to safely and reliably obtain samples for research or microbiology purposes in thoracic surgery patients.

1. Huang Y-C T, Bassett MA, Levin D, Montilla, Ghio AJ. Acute Phase Reaction in Healthy Volunteers After Bronchoscopy with Lavage. Chest 2006; 129 (6): 1565-9.

2. Terashima T, Amakawa K, Matsumaru A et al. BAL Induces an Increase in Peripheral Blood Neutrophils and Cytokine Levels in Healthy Volunteers and Patients with Pneumonia. Chest 2001; 119 (6): 1724-1729.

\section{P112 THERAPEUTIC WHOLE LUNG LAVAGE FOR SILICOSIS - FIRST APPLICATION IN THE UK}

doi:10.1136/thoraxjnl-2012-202678.395

'B Prudon, ${ }^{2} \mathrm{R}$ Attanoos, ${ }^{3} \mathrm{C}$ Morgan, ' $\mathrm{SC}$ Stenton. 'Department of Respiratory Medicine, Newcastle upon Tyne Hospitals NHS Foundation Trust, Newcastle upon Tyne, UK; ${ }^{2}$ Department of Histopathology, University Hospital Llandough, Cardiff, UK; ${ }^{3}$ Department of Anaesthetics, Royal Brompton \& Harefield NHS Trust, London, UK

Silica is a highly fibrogenic dust and this is reflected in the low amounts of dust found in the lungs of those with fatal silicosis approximately $3 g$ in total. Despite a low workplace exposure limit for respirable silica, new cases of silicosis continue to be diagnosed. There is no effective pharmacological treatment. In China, whole lung lavage (WLL) has been performed for silicosis with evidence of improved dyspnoea at 6 months. To our knowledge the technique has not previously been attempted in the UK.

We carried out WLL in 2 stonemasons with silicosis. Patient A presented aged 41 in 2007 with MRCP dyspnoea score 3, FVC 3.27L ( $60 \%$ predicted), and radiographic features of extensive silicosis (Category 3R, ILO classification). To determine if mineral could be removed from his lungs, a bronchoscopic lavage was performed using $180 \mathrm{ml}$ saline. The lavage fluid contained $4.8 \mathrm{~g} / 1$ of mineral. WLL was then performed with 7L saline on the right, and a month later $12 \mathrm{~L}$ on the left. Each procedure lasted approximately 1 hour and the patient was discharged without complication within 24 hours. The washings contained $0.66 \mathrm{~g} / 1$ mineral. The total removed was approximately $7.9 \mathrm{~g}, 50 \%$ of which was silica, (silica content was determined by transmission electron microscopy with energy dispersive x-ray spectrometry). On review at 6 months there had been no clinical or radiological changes.

Patient B presented aged 31 with MRCP dyspnoea score 2, FVC $3.96 \mathrm{~L}(65 \%$ predicted), and radiographic features of silicosis (Category C). A 9L right WLL produced considerably less mineral $(0.09 \mathrm{~g} / \mathrm{l}$ : total approximately $0.1 \mathrm{gm}) .21 \%$ was silica.

These cases demonstrate that WLL is acceptable and safe in patients with silicosis in the UK, and that substantial quantities of mineral can be removed from the lungs. It is postulated that reducing the silica burden will slow the rate of disease progression, but there is no evidence in support of that. Evidence will be difficult to adduce given the variable nature of silicosis and its relatively slow rate of progression. In the meantime, we suggest that WLL is considered for younger patients with advanced silicosis.

\section{P113 SECRETED LYSYL OXIDASE IS ELEVATED IN THE BRONCHOALVEOLAR LAVAGE FLUID OF PATIENTS WITH IDIOPATHIC PULMONARY FIBROSIS}

doi:10.1136/thoraxjnl-2012-202678.396

${ }^{1} \mathrm{CJ}$ Calderwood, 'MG Jones, ${ }^{2} \mathrm{~L}$ Hoile, ${ }^{2} \mathrm{~T}$ Havelock, ${ }^{3} \mathrm{TM}$ Maher, ${ }^{2} \mathrm{KMA}$ O'Reilly, ${ }^{1}$ DE Davies. 'University of Southampton, Southampton, United Kingdom; 'Southampton University Hospitals NHS Trust, Southampton, United Kingdom; ${ }^{3}$ Royal Brompton Hospital, London, United Kingdom

Background Idiopathic pulmonary fibrosis (IPF) is a chronic progressive fibrotic lung disease for which there are no effective treatments. As a result the prognosis is poor, with a median survival of 3 years from the onset of symptoms. The key feature of IPF is formation of 'fibroblastic foci', accumulations of highly active, proliferative fibroblasts and myofibroblasts that lay down vast quantities of collagen and other extracellular matrix proteins. Lysyl oxidase (LOX) is a secreted enzyme involved in cross-linking of collagen and implicated in several fibrotic conditions. Increased cross-linking due to LOX upregulation in IPF may contribute to decreased lung compliance. Further, LOX may represent a biomarker that can be used to detect early responses or 'proof-of-mechanism' in clinical trials for new IPF drugs. We hypothesized that levels of LOX protein were higher in bronchoalveolar lavage (BAL) fluid from IPF patients compared to healthy controls.

Methods BAL fluid was collected from patients with IPF or volunteers following ethical approval and informed consent. A quantity of BAL fluid determined to contain $20 \mu \mathrm{g}$ protein was concentrated with Strataclean resin and resuspended in $2 x$ sample buffer. LOX protein content was determined by SDS-PAGE and western blotting followed by densitometry.

Results LOX can be detected in BAL fluid from patients with IPF. Both pro and active forms of the LOX enzyme are significantly elevated in IPF compared to healthy controls, with active LOX detected in $12 / 25$ IPF patients compared to $1 / 9$ healthy controls.

Conclusions There is increasing evidence for a role of LOX in fibrosis. Here we suggest LOX may be involved in IPF pathogenesis, and demonstrate that it is possible to detect secreted LOX in BAL fluid from patients with this condition. LOX may therefore represent a biomarker that could be used in clinical trials for IPF. Further work would be required to validate and optimise assays for clinical use.

\section{P114 PULMONARY FUNCTION PROGRESSION IN LANGERHANS CELL HISTIOCYTOSIS}

doi:10.1136/thoraxjnl-2012-202678.397

SR Krivinskas, A Chu, PW Ind. Imperial College Healthcare Trust, London, England

Langerhans Cell Histiocytosis (LCH) is a rare, dendritic cell disorder commonly involving the lungs either alone, (PLCH) or as part of multi-system disease (MSLCH). The natural history is variable, ranging from spontaneous resolution to progressive respiratory failure and death. Until recently there were no large follow up series containing lung function data (Tazi A et al, ERJ 2012; 02107-2011)

We retrospectively compared pulmonary function over time in patients with PLCH and in MSLCH with lung involvement from our database of 83 adult LCH patients. 46 patients were male; mean age at diagnosis was 34 (range 16-76) years (y). 9/83 patients, 4 male, mean age $32 \mathrm{y}$, had PLCH. 21/83 13 male, mean age $28 \mathrm{y}$, had MSLCH with lung involvement.

All PLCH had smoked with mean 14 pack-y. 7 continued smoking after diagnosis. Initial lung function $(n=8)$ at a mean of $1(-1$ to 